\title{
Summary and concluding remarks
}

\$1 This book has explored several paths toward studying the relation between science and language, especially the Latin language - the leading language of science and learning for the longest time. Scientific Latin is the far too littlestudied connecting element between Greek science in Antiquity and contemporary modern science in what could be called a translatio linguae (chap. 16 §3). Latin had to face problems in vocabulary, syntax, and style in order to express and appropriate Greek scientific thought. Although a radical dependence of thought on language as advocated by Whorf and others certainly goes too far - after all, human beings do share a body with a more or less identical sensorium grounding our experience in the world - the more remote from direct sensation ('abstract') things get, the more room for differences among peoples and languages emerges. As most scientific languages in the history of mankind have been in contact with one another and have influenced each other decidedly (as has been described for Latin from Greek, then the European vernaculars from Latin), it is not trivial to ascertain how far divergence in scientific expression is feasible. In this book, it has become apparent several times that Greek, Latin, German, and English have produced somewhat different 'flavours' of science. Our modern European scientific languages strongly depend on Latin; indeed, they are full of Latin loanwords and calques, especially so in the case of English, in which language $57 \%$ of the vocabulary in one sample ultimately derived from Latin, either directly $(28.24 \%)$ or through French $(28.30 \%) .{ }^{1}$ For instance, English very often uses Latin to derive adjectives from Germanic nouns: 'star' $\rightarrow$ 'stellar', 'heart' $\rightarrow$ 'cardiac', and so on. ${ }^{2}$ Such strata from prestigious languages of learning for cultivated concepts are frequent in many cultural spheres: Hindi or Thai tend to use Sanskrit words similarly to how English uses Latin ones. The strong influence of Latin on modern scientific English will have become palpable throughout this study. What follows summarises the main results of the book.

\$2 Part 1 developed the following matters by working back from the present; here they are summarised chronologically. The semantics of 'science' were studied, as well as the question of whether premodern times had similar concepts and how different languages have called and still call such activities. The concept 'science'

1 According to the computerised survey of about 80,000 words in the Shorter Oxford Dictionary (3rd ed.) by Finkenstaedt \& Wolff (1973).

2 On this topic, see the interesting article https://en.wikipedia.org/wiki/Latin_influence_ in_English (December 2018), providing many examples. In similar circumstances, German uses compounds instead: 'stellar nebula' = Sternnebel, 'cardiac attack' = Herzinfarkt.

Ә Open Access. ( 2021 Philipp Roelli, published by De Gruyter. (C)BY This work is licensed under the Creative Commons Attribution 4.0 International License.

https://doi.org/10.1515/9783110745832-026 
turned out to differ somewhat between languages and through time since Classical Greek, which is hardly surprising: the same is true for most abstract notions (think of 'art', 'religion', 'nation', or 'family'). Nonetheless, this study has hopefully shown convincingly that science goes back organically to scientific activities undertaken in Greek Antiquity, which in turn owe their existence to a unique combination of several factors in classical Greece, most importantly: natural-philosophical speculation, the sophist movement analysing language, and Aristotle and his school. Although many similar factors can be observed in cultures before and alongside the Greeks, their combination into a durable way of thinking (Fleck's Denkstil) recognisably similar to our 'science' emerges only with the Greeks in the time around $500 \mathrm{BC}$ (chap. 7 §9). These factors led to a demand for stronger requirements to make explanations credible and certain, such as mathematical proof, precise observation, but also a scrutiny of (the Greek) language and its precise form of assertions and of logic (chap. 7).

Although many sciences were begun in classical and Hellenistic Greece, Classical Greek did not at first have a single word to denominate 'science' as a whole. Plato and Aristotle mostly used غ̇лı able to man (in the plural already denoting scientific disciplines for Aristotle), although for mathematical sciences $\mu \alpha \dot{\theta} \eta \mu \alpha$ was also used. The prime activity

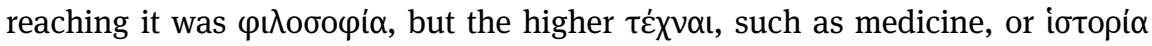
also strove for maximal attainable certainty. In Hellenistic and Roman times, the

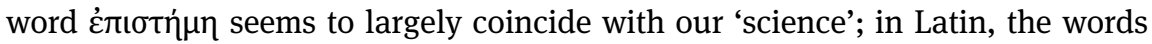
scientia and disciplina, and in some contexts ars (especially as artes liberales), were its usual renderings. Which one was chosen depended largely on the author. Only in the High Middle Ages did the first option become the standard term (helped by the Arabic ' $i l m$ ), whereas the second developed more and more toward our 'discipline' as a single scientific branch or, in fact, a branch of other activities as well. It was shown (1.3) that for most pertinent words in Greek, Latin chose a single equivalent, a kind of interpretatio Romana of Greek epistemology.

Historians of science have proposed vastly different definitions or characterisations of what science is. Many of their criteria were found not to be applicable to diachronic samples of science. Some were reviewed, and a few were found that characterise science in a similar way to the following criteria. In order to be able to tell science apart from other activities through this entire time span, a list of criteria was proposed that scientific study needs to fulfil. The first three items were treated as necessary, the second three as additional characteristics that may be missing in some cases: (I) a systematic method and well-defined topic, (II) finding patterns and explaining them step-by-step, (III) an unbiased seeking of confirmation or refutation, (IV) coherence and non-sterility, (V) a community effort, and (VI) formalisation of the results. 
These points together lend themselves to a specific, 'scientific' Denkstil. Points (II) and (III) together provide the typical combination of a general and theoretical explanatory framework and the gathering of trustworthy data to check and rein it in. Among such approaches, science must take into account what may be called 'generally acknowledged facts' (alluded to in points II and III). Science using criteria II and III to progress further (using these 'legs' to walk, as Galen put it) will produce new such generally acknowledged facts, thus accelerating its own development. Since these are fallible (like everything human), a revolutionary component joins the evolutionary one, occasionally leading to breaks in scientific development. Within these rather general characteristics, many different subDenkstile are possible, and the sciences have, indeed, a tendency to continuously evolve their Denkstil; Fleck clearly showed that this does not entail a linear progress toward 'truth', but it also seems clear that the more generally acknowledged facts there are, the less freedom for Denkstile within a science.

Point VI, 'formalisation', is conducive to a special kind of language, for which the following criteria were tentatively put forward: (i) well-defined terminology, (ii) exactness and unambiguity, (iii) extendability and flexibility, (iv) perspicuity, and (v) evidentiality and modality. Together, they constitute requirements for a certain kind of technical language. It seems clear that the more a science advances, the more technical its language will become, as it has to cover more and more ground and gets further and further from the everyday matters for which everyday language is tailored. If there is a more or less autonomous and sizeable community of researchers (point $\mathrm{V}$ ), they will automatically develop their own technical jargon.

The above criteria have not restricted scientific study to 'nature'. Indeed, it was found that this restriction is a recent acquisition of the English language that is not shared by most other European languages used for comparison (chap. 1). Thus, today, philology, linguistics, sociology, historiography, law, and other fields that were traditionally seen as scientific are usually no longer called 'sciences' in English. The term now most often used for them is 'scholarship'. This significant and unilateral change in English, which was shown to become clearly noticeable only toward the end of the nineteenth century, was not followed in the present study, and 'science' was treated in its original wider meaning covering fields of activities delineated by the above criteria. It was pointed out that this tendency of the English language might be linked to the Royal Society's exclusion of human sciences and theology from its studies in the late seventeenth century in order not to get caught up in Reformation quarrels (chap. 14 §3). Due to this special development of English, contemporary English-language histories of science have a tendency to be histories of natural science and to produce a skewed view of all the activities that were seen as scientific in their time and as belonging together as such. 
\$3 Part 2 provided a panorama based on Latin primary texts in order to illustrate the historical development and exemplify the language and Denkstil used among Latin scientific authors. The first chapter pointed out that the language of science depends on the scientific genre used. It was noted that some forms of scientific communication important in Greek and Latin times, such as didactic poetry, dialogues on science, or letters between scientists, have fallen quite out of use in more recent times. A further difference is whether texts were written by specialists for specialists, with didactic purposes for future scientists, or for a wider public. It has been stressed that in order to compare scientific language, if possible, care should be taken to make comparisons within the same textual genre. After these preliminaries, a chronological discussions began with a brief look at Latin's rôle model, Greek (chap. 7). Then, epochs of Latin science were proposed and important authors and texts briefly presented. It became clear that the usual epochs (Antiquity, Middle Ages, modernity) do not work well for science. Instead, the following divisions were proposed and described; they alternate between a relative stability of scientific approach and times of revolutionary change. The foundations of Roman science were laid by imports from Greek roughly from 100 BC to AD 200 and by the growth of the genuinely Roman science of jurisprudence (chap. 8), followed by an approach mostly followed by Christians which was centred on encyclopaedism and soon acquired the Platonic classification of the sciences into the artes liberales (chap. 9); it lasted from Late Antiquity through the Early Middle Ages, and was of a decidedly didactic and conservative nature. Significant novelty in many fields, as well as new impulses from newly available Greek and Arabic texts, marked an important caesura in the long twelfth century (chap. 10). The Greek scientific Denkstil only fully arrived in Latin in this period the turning point of Western European intellectual history. A variety of approaches were developed by different people, some of which led (chap.11) to scholastic university science with a very distinctive and standardised kind of Latin which developed at the new universities with their emphasis on disputation, dialectic, and logic. Early modern times (chap. 12) brought new approaches again, but most of them, especially the humanist and the hermetic ones, were rather dead ends for science, and the Renaissance can be seen rather as a time of stagnation or even regress in scientific methodology with its often rigourist, classicist approach to Latin. But 'scholastic' science kept being widely practised through the Renaissance at many universities and was enriched by more experimental magia naturalis. The Scientific Revolution (chap. 13) brought more important and lasting changes to scientific methodology in the later sixteenth and seventeenth centuries which quite justify the label. Again, a variety of new methods were proposed and used with different degrees of success in various fields. The most successful novelty was certainly a stronger use of mathematics (which over the past few cen- 
turies had started to produce the necessary foundations) and of a methodical use of experimentation. This led to a kind of science that started to actively 'mine' new generally acknowledged facts, enabling it to accelerate its development considerably. Latin was still the dominant language of science throughout the Scientific Revolution, a Latin much closer to 'useful' scholastic Latin than to 'beautiful' humanist Latin. The international aspect the movement had thanks to Latin helped it gain a momentum that would hardly have been possible had everyone already used their own vernaculars. Chapters 14 and 15 studied Latin's demise and scientific niches where Latin kept being used beyond the eighteenth century respectively. The main culprit of Latin's demise was found to have been French nationalism. The most enduring niche of scientific Latin can be found in Jesuit schoolbooks of the first half of the twentieth century. Some consequences of the change of linguistic medium from Latin to vernacular science concluded this part of the book (chap. 16).

\$4 Part 3 studied scientific Latin linguistically from several angles, mostly numerically or comparatively. Corpus Corporum, an open meta-corpus of Latin texts developed for this study, was used in most cases. After an introduction detailing what is known about scientific English (chap. 17), the first three chapters (chaps 18-20) used corpus linguistic methods to study scientific Latin, especially the differences in its PoS composition and syntax. Five large benchmark corpora for different periods made up of prose texts of all kinds were used as a basis for comparison. They were compared with a general group of forty more theoretical scientific prose texts (chap. 18), then with four more thematic scientific samples: an arithmetic sample was studied in some depth, as were samples of historiography, of scientific poetry (chap. 20), and of medical texts, representing less strict or less theoretical science (chap. 21). Some further out-groups were compared to the general sample: poetry, the Vulgate, the Digesta, a collection of mediaeval charters, and some scientific translations from Greek. The corpus linguistic findings are summarised in chapter $20 \$ 8$. Some parameters known to be specific to scientific English - the use of non-conjugated verb forms, prepositions and conjunctions, the passive voice, a lack of the first person singular, and nominalisation of processes - were tested for Latin as far as they were applicable. Some of them were found to be also typical for scientific Latin (high numbers of PREP, 3rd PAS; low numbers of 1st SG, POSS), but others were not (CONJ, non-finite verb forms); moreover, some seem to be typical for scientific Latin but not for scientific English, such as high numbers of ADJ. Some are typical for Latin science but not directly comparable to or not yet studied for English (high numbers of NOM case, the verb ESSE, suffixation; low numbers of ablativus absolutus), some seemed typically Greek (sentence particles), and some opened up new questions: low en- 
tropy (hitherto not studied for scientific texts). Principal component analysis and stylometry were employed to plot the texts in two-dimensional space and in tree form respectively, based on parts of speech and similar parameters. It proved possible to identify quite clear-cut groups of types of Latin; they were compared to those observed in part 2. The results are summarised in chapter 19; they were labelled as (i) hexametric, (ii) rhetorical, (iii) plain, (iv) bombastic, (v) scholastic, (vi) mathematical, and (vii) modern academic Latin. It became clear that due to Latin's deep memory, these approaches and their respective styles of writing did not supplant one another for good: for instance, Vesalius could still use a rather rhetorical approach in early modern times. Nonetheless, it seemed that after the great watershed of the long twelfth century, only the last three approaches would be used for serious scientific communication among experts in the more theoretical sciences (which one of them, depended on the field). Whereas the rhetoric approach in science can be traced back to Plato, the scholastic one to Aristotle, and the mathematical one to Euclid, the final 'modern academic' style looks like a summa of the earlier styles and lacks a clear Greek model - a Latin that would have been very well suited as an international scientific auxiliary. For political reasons this did not happen. Before the long twelfth century, Latin science was nearly exclusively popular science or practical science. It was shown that the language of an example of the latter, medicine, was quite similar to the 'plain' approach of Pliny (chap. 21), also and even especially after the Middle Ages. Scholastic and mathematical texts clustered furthest away from the non-scientific benchmark samples together with translations from Greek. Poetry and the Vulgate were also far off, but in opposite directions. For the arithmetic texts (chap. 20), groups within the sample were less clear-cut, but the sample as a whole differed strongly from normal Latin; mathematically minded authors from the general science sample (Copernicus, Newton) clustered closest to it. It seems that the language of these texts remained rather constant and closely linked to that of Euclid (the first verbatim Latin translation of him ended up close to the root of all subsequent arithmetic texts). The texts from the eighteenth century and later tended to cluster somewhat apart. The language of historiography was found to differ quite strongly from the more strictly scientific texts, but not to differ much from non-scientific Latin; that of scientific poetry was, as expected, found to share some characteristics with other poetry and some with prose science.

Different approaches were chosen for the remaining chapters. Chapter 21 studied how new phenomena were named by Latin medical writers in different times. It found a great dependence on Greek and, from early modern times onward, formalised systems of, for instance, different Latin suffixes for different kinds of ailments. In the mathematical sciences, formalisation went even further, 
a step that can hardly be underestimated in its importance for the development of modern much more formalised forms of science that are thus much less dependent on natural language - a development that within 'richer' Greek might not have seemed necessary. The abundance (or dearth) of a language's linguistic tools may thus be secondary to the desire within a community of speakers of a language to express certain kinds of thought. If such a desire is present and there is time, it will find its ways of expressing itself. This is very well illustrated by the fact that even minor languages have in the past two centuries acquired the means to discuss scientific topics of nearly any kind - notwithstanding that this happened and still happens largely by borrowing structures from the major scientific languages of Latin, French, German, and English. Indeed, English and German have borrowed so much of their structure and vocabulary from Latin that not only will French and the Romance languages have to be called Latin's daughters, but the Germanic languages can very fittingly be called its stepdaughters. ${ }^{3}$ Above (chap. 16 §), it was stressed how much vocabulary and even syntax was taken over from Latin to form Germanic standard languages that could be used in all contexts, including the scientific. The next two chapters, consequently, compared how Graeco-Latin scientific terminology was adopted by other languages: by traditional ones (chap. 22) and by contemporary ones (chap. 23). Different approaches have become clear: Sanskrit packed much information into compounds, Chinese invented new combined characters in its flexible writing system, and Arabic often used circumlocutions. Among the European languages, some have just taken over Latin terms (the Romance languages, English), some have made calques (Icelandic), some sometimes the former and sometimes the latter (German, Russian), some have only borrowed Greek-based terms and adapted Latin-based terms (Modern Greek), and some have used loanwords (Indonesian).

Chapter 24 sought to synthesise the relationship between science, culture, and language. It was argued that science, as defined in chapter 4, is a Greek Denkstil that can be found at best in nuce outside the Greek cultural horizon. Mesopotamian and ancient Egyptian candidates for science, as well as some from India and China before intense Greek contact, were briefly compared. Then the typical language used in scientific Greek and its translation into Latin was tackled. The three potentially most important linguistic features for scientific Greek expression were considered: nominalisation using the article, free compounding (both hard to translate into Latin), and new terms made by suffixation (typical for scholastic Latin). Nonetheless, and not withstanding that it took Latin a long time until science started to be done seriously in this medium, in the Late Middle Ages and

3 As Leonhardt (2013: 37) proposed. 
early modern period Latin fulfilled the rôle of lingua scientiarum very well indeed. In order to do so, it made use of various devices, such as creating new Latin terms by suffixation or the formalisation of thought in many fields, especially mathematics, logic, and scholasticism, thus taking over, but slightly adapting, the Greek scientific Denkstil.

§5 By virtue of being the linguistic medium of the Scientific Revolution, and through the widespread adoption of its scientific vocabulary, Latin can be said to lie directly at the roots of modern science (whereas Greek does so more indirectly), and in fact in a sense it lives on in modern scientific languages (chap. 23), especially in English. Not only does English take very much of its scientific vocabulary from Latin (both directly, and indirectly through French) but it follows many of its structures, such as -ing-form clauses in the function of the Latin participium coniunctum and ablativus absolutus (chap. 16 §2). This can be seen as standing in a certain contrast to German, which was in many fields the leading language of scientific communication from the middle of the nineteenth century until Germany's defeat in the two World Wars. German functions much more like Greek; in many fields, full advantage was taken of its ease of compounding words and deploying complex syntactic structures, which may be best seen in its philosophers and human scientists, whose texts are often nearly untranslatable into English. Such compounds are often Augenblicksbildungen, ${ }^{4}$ something strongly disliked by Latin and English. In Latin this tendency was weakened by scholasticism, which lost much of Latin's inhibitions in coining new words, but humanism tried to check this development, in some sciences with more success, in others less. Contemporary English science can be seen as driving this isolating approach even further: formulas and Graeco-Latin technical terminology that is often subsequently used only as acronyms abound today; Augenblicksbildungen are not used. This study has thus, in passing, demonstrated differences in the scientificolinguistic approach in different languages, especially in Greek, Latin, English, and German, and revealed a certain congeniality of Greek and German on the one hand and Latin and English on the other. Clearly, this is a field that would need a lot more research and that does, finally, seem to be attracting more attention. The hegemony of English as the vehicle of science may be seen as a kind of revival of the Latin way of formulating science in language, although with the use of for-

4 We have met many such cases that were hard to translate into English in the passages quoted in this book: Erkenntnisarbeit, Methodenzwang, Normenentfaltung, voraussetzungsloses Beobachten, or sachliches Wissenwollen. Such compounds may continue to be used in their fields or they may not; in either case, their first use is seen as something natural in German scientific language with no need of justification or even definition. 
mulas and less dependence on linguistic structures, Anglo-Saxon science has become more technical and less philosophical, one might say. The general scientific Denkstil, however, seems to have been largely preserved in the transition from Latin to the European vernaculars and especially to English today. The English of many contemporary natural sciences has reached a degree of 'ugliness' - rhetorically speaking - that the 'worst' scholastic Latin writer could hardly have managed in Latin, which may show that the scholastics were on the right track in putting content before beauty in science. In conclusion, the fixed language Latin can therefore be said to be not at all dead, but thriving and in full vigour in scientific English today. 